\title{
Prospective, multicenter study of pleural adhesion in repeated pulmonary surgery
}

\author{
Yoshihiro Miyata ${ }^{1}$, Hiroshi Date ${ }^{2}$, Mitsugu Omasa ${ }^{2}$, Kenji Suzuki $^{3}$, Kazuya Takamochi $^{3}$, Seiki Hasegawa ${ }^{4}$, \\ Nobuyuki Kondo ${ }^{4 *}$, Morihito Okada ${ }^{1}$ \\ From World Society of Cardiothoracic Surgeons 25th Anniversary Congress, Edinburgh \\ Edinburgh, UK. 19-22 September 2015
}

\section{Background/Introduction}

Pleural adhesion (PA) complicates repeated pulmonary surgery.

\section{Aims/Objectives}

Herein, we prospectively investigated the degree of PA in the second thoracotomy to assess the impact of the previous ipsilateral thoracic procedure.

\section{Method}

Seventy patients, with a median age of 67 years, undergoing a second thoracotomy with complete medical records of the previous thoracotomy from 4 institutions were included in this study. The mean interval from the first operation to the second operation was 20 months (1-105 months). The site and the extent of adhesion as well as duration and amount of bleeding while dissecting the adhesion were recorded.

\section{Results}

Fifty-four (76\%) patients had PA to the chest wall and $10(14 \%)$ had dense and total PA in the thoracic cavity. Patients with PA experienced more bleeding (215 vs. $29.3 \mathrm{~g}$ ), a longer drainage period (4.7 vs. 2.3 days), and higher post-operative morbidity ( $28 \%$ vs. $6 \%$ ) than those without PA in the second operation. According to ROC analysis, cut-off values for operation duration, amount of bleeding, and length of thoracotomy incision in the initial operation for predicting PA were 75 minutes, $10 \mathrm{~g}$, and $6.0 \mathrm{~cm}$, respectively. On the basis of the univariate analysis for PA, operation time $>75$ minutes, bleeding $>10 \mathrm{~g}$, thoracotomy incision length $>6.0 \mathrm{~cm}$, and segmentectomy or lobectomy in the initial surgery were significantly associated with PA. Multivariate analysis revealed only thoracotomy incision length $>6.0 \mathrm{~cm}$ was the independent predictor for PA $(p=0.0065)$. All patients with thoracotomy incision length $>6.0 \mathrm{~cm}$ showed PA, but $54 \%$ of patients with thoracotomy incision length $<6.0 \mathrm{~cm}$. On the other hand, thoracotomy incision length was not associated with dense and total PA. Multivariate analysis identified only post-operative drainage period $>5$ days in the initial surgery to be the independent predictor for dense and total PA in the thoracic cavity $(\mathrm{p}=0.016)$.

\section{Discussion/Conclusion}

Long thoracotomy incision length in the initial surgery is a predictor for PA at the second surgery. Dense and total PA might be caused by post-operative inflammation in the thoracic cavity due to prolonged air leakage.

\section{Authors' details}

'Surgical Oncology, Hiroshima University, Hiroshima, Japan. ${ }^{2}$ Kyoto University, Kyoto, Japan. ${ }^{3}$ Juntendo University School of Medicine, Tokyo, Japan. ${ }^{4}$ Hyogo College of Medicine, Mukogawa, Japan.

Published: 16 December 2015

doi:10.1186/1749-8090-10-S1-A343

Cite this article as: Miyata et al:: Prospective, multicenter study of pleural adhesion in repeated pulmonary surgery. Journal of Cardiothoracic Surgery 2015 10(Suppl 1):A343.

${ }^{4}$ Hyogo College of Medicine, Mukogawa, Japan

Full list of author information is available at the end of the article 